\title{
Gap junction $\beta$-2 expression is negatively associated with the estrogen receptor status in breast cancer tissues and is a regulator of breast tumorigenesis
}

\author{
ABHIJITH SHETTAR ${ }^{1}$, SUREKHA DAMINENI ${ }^{1}$, GEETASHREE MUKHERJEE ${ }^{2,3}$ and PATURU KONDAIAH ${ }^{1}$ \\ ${ }^{1}$ Department of Molecular Reproduction, Development and Genetics, Indian Institute of Science, Bangalore, \\ Karnataka 560012; ${ }^{2}$ Department of Pathology, Kidwai Memorial Institute of Oncology, Bangalore 560029 Karnataka, India
}

Received July 11, 2017; Accepted June 13, 2018

DOI: $10.3892 /$ or.2018.6764

\begin{abstract}
Gap junction $\beta-2$ gene (GJB2, also known as connexin 26) is a member of the connexin family which forms gap junction channels. Many connexin genes have been considered to be tumor suppressor genes. However, the overexpression of GJB2 has been found to be associated with a poor prognosis in several human cancers. In our previous microarray study, we revealed the overexpression of GJB2 in breast cancer tissues. Hence, in this study, we investigated the expression of GJB2 in human breast cancer and its role in breast cancer cell proliferation and migration. The RT-qPCR results revealed the upregulation of the GJB2 gene in invasive ductal carcinoma $(\mathrm{P}<0.001)$ of the breast. Immunohistochemical analysis revealed an intense cytoplasmic and membrane staining. We observed that the staining for GJB2 was more intense in the majority of the estrogen receptor (ER)-negative breast cancer tissues compared to the normal breast tissues $(\mathrm{P}<0.0001)$. By contrast, the majority of the ER-positive breast cancer samples exhibited weak to moderate staining; however, this difference was not statistically significant compared to the normal tisues. The knockdown of GJB2 in human breast cancer cell lines using shRNA led to a significant decrease in the proliferative ability and an increase in the migratory ability of breast cancer cells. In addition, the knockdown of GJB-2 led to a significant reduction in tumor volume and proliferation (as demonstrated by MIB-1 staining) in orthotopic xenografts in immunocompromised mice. On the whole, the findings of this study indicate that GJB2 may be an important regulator of breast tumorigenesis.
\end{abstract}

Correspondence to: Professor Paturu Kondaiah, Department of Molecular Reproduction, Development and Genetics, Indian Institute of Science, CV Raman Road, Bangalore, Karnataka 560012, India E-mail: paturu@iisc.ac.in

Present address: ${ }^{3}$ Department of Histopathology, Tata Medical Center, Rajarhat, Kolkata 700160, India

Key words: connexins, gap junction $\beta-2$, estrogen receptor, breast cancer

\section{Introduction}

The intercellular communication and passage of small molecules between neighbouring cells is mediated by gap junctions, which play a variety of important roles in various biological processes, including cell differentiation, homeostasis, morphogenesis and growth control (1-3). Connexins have been considered as tumor suppressors in many types of cancer (4-6). However, recent evidence indicates the involvement of connexins in promoting cell proliferation (7), regulating apoptosis (8), and promoting chemoresistance (9), migration (10), regulation of epithelial-mesenchymal transition (EMT) (11), tumor differentiation and angiogenesis (12-15).

Gap Junction $\beta$-2 (GJB2, also known as connexin 26) is one of the members of 21 connexin family genes that form the gap junction channels, which is abundantly expressed in the skin, liver and breasts. A previous study indicated an association of the mutations in the GJB2 gene with neurosensory deafness (16). Previous studies have also revealed the overexpression of GJB2 in several carcinomas, including head and neck, colon, prostate and skin tumors (17-21). In breast cancer, GJB2 has been indicated to be a tumor suppressor due to its very low expression in breast tumor tissues, perhaps due to hypermethylation (22). By contrast, there are also studies indicating the overexpression of GJB2 in breast cancer tissues and its involvement in metastasis to the lymph node and its association with a poor prognosis $(23,24)$. A high expression of GJB2 has also been detected in $>50 \%$ of invasive breast cancer tissues when compared to normal human breast tissues. In addition, the expression of GJB2 has shown to be associated with a poor prognosis of lung and esophagus carcinoma (25-27). Collectively, these studies support a pro-tumorigenic role for GJB2.

In view of these findings, we hypothesized that GJB2 may be associated with the hormonal status of breast tumors, which may be one of the reasons for the discrepancy in the reported role of GJB2 in breast cancer. In the present study, we examined the expression of GJB2 in breast cancer tissues of various hormonal statuses and investigated the role of GJB2 by knocking down its expression in breast cancer cells followed by in vitro and in vivo experiments. Our data revealed a negative association of GJB2 protein with the ER status of breast 
tumor tissues. Furthermore, GJB2 was found to be involved in the growth of breast tumors.

\section{Materials and methods}

Patients and tumor samples. Breast tumor tissue biopsy samples as well as adjescent normal breast tissue biopsy samples were obtained from breast cancer patients who presented at the Kidwai Memorial Institute of Oncology (KMIO) Bangalore from June, 2009 to March, 2012. For the present study, a total of 99 breast tumor samples and 46 tumor adjescent normal samples were collected. All patients provided informed consent following the approval of the Kidwai Memorial Instititue of Oncology (KMIO) Medical Ethics Committee. The status of estrogen receptor (ER), progesterone receptor (PR), HER2/neu and pathological data, including tumor grade, size and lymph node status were obtained from the pathology records of the respective patients

Cell lines and cell culture. The breast cancer cell lines, BT-474 and MCF7 (ER-positive), were obtained from the American Type Culture Collection (ATCC, Manassas, VA,USA) and were cultured in Dulbecco's modified Eagle's medium (DMEM, high glucose; Sigma-Aldrich, St. Louis, MO, USA) supplemented with $10 \%$ fetal bovine serum (FBS; Invitrogen/Thermo Fisher Scientific, Inc., Waltham, MA, USA), penicillin $(1 \mathrm{kU} / \mathrm{ml})$ and streptomycin $(0.1 \mathrm{mg} / \mathrm{ml})$. The cells were maintained under standard culture conditions at $37^{\circ} \mathrm{C}, 5 \% \mathrm{CO}_{2}$ in a humidified incubator (Thermo Fisher Scientific, Inc.).

Reverse transcription-quantitative PCR (RT-qPCR). RNA was extracted from the tissues/cells using TRIzol reagent (Sigma-Aldrich) according to manufacturer's instructions. For quantitative (real-time) PCR analysis, total RNA was reverse transcribed using a cDNA synthesis kit (Applied Biosystems, Foster City, CA, USA). qPCR was performed in triplicates using Dynamo ${ }^{\mathrm{TM}}$ SYBR-Green $2 \mathrm{X}$ mix (Finnzymes; Thermo Fisher Scientific, Inc.) on an ABI Prism 7900HT sequence detection system and analyzed with SDS 2.1 software (both from Applied Biosystems). The expression of TBP was used for normalization. The expression of TBP was consistent across the breast cancer tissues and adjacent normal tissues in the microarray experiments. For the normalization of gene expression in the cell lines, RPL35A was used. The analysis was performed using SDS 2.1 software (Applied Biosystems). The fold change in the levels of the mRNA was calculated from the data using the $2^{-\Delta \Delta C t}$ method (28). For analyzing the gene expression of GJB2 in breast tissue samples, a set of 50 invasive breast cancer tissue samples and 25 adjacent normal samples were used.

The sequences of the primers used for PCR in the present study were as follows: GJB2 forward, 5'-AGCGCAGAGACC CCAAC-3' and reverse, 5'-GGTGGAGTGTTTGTTCAC-3'; TBP forward, 5'-GATCAGAACAACAACAGCCTGCC-3' and reverse, 5'-TTCTGAATAGGCTGTGGGGT; RPL35A forward, 5'-GGGTACAGCATCACTCGGA-3' and reverse, 5'-ACGCCCGAGATGAAACAG-3'.

Immunohistochemistry. For histological analysis, a different set of breast tumor tissues were obtained from blocks archived at the Department of Pathology at the Kidwai Memorial Institute of Oncology (KMIO). Tissue sections (5- $\mu \mathrm{m}$-thick) from the paraffin-embedded tumor specimens were collected on silane-coated slides and immunohistochemistry for GJB2 was performed on 49 tumor tissue sections (27 ER-negative and 22 ER-positive) and 21 adjacent normal tissue sections. Antigen retrieval was performed by heat treatment of the deparaffinised sections in citrate buffer (10 mM; pH 6.0). Following the initial processing steps, the sections were incubated overnight with goat polyclonal anti-GJB2 antibody (1:100 dilution; ab59020; Abcam, Cambridge, MA, USA), at $4^{\circ} \mathrm{C}$. This was followed by incubation with the supersensitive non-biotin horseradish peroxidase detection system (QD440-XAK, Biogenex, Fermont, CA, USA). In addition, 3,3'-diaminobenzidine (Sigma-Aldrich) was used as the chromogenic substrate.

Evaluation of immunohistochemistry results. The immunohistochemistry scoring for the expression of GJB2 was based on a semi-quantitative scoring method as previously described from our laboratory (29), where both the intensity and percentage of cells with positive staining were analyzed by experienced pathologists and a combined score was obtained. The combined score was calculated by the multiplication product of both scores. The scores were as follows: i) percentage of cells: no staining, $0 ; \leq 10 \%$ or less of cells stained, $1 ; 11-50 \%$ of cells stained, 2 ; and $\geq 50 \%$ or more of cells stained, 3 ; and ii) intensity: no staining, 0 ; weak staining, 1 ; moderate staining, 2 ; and strong staining, 3 . Thus, the combined scores ranged from 0-9. Only scores from 4-9 were considered positive for staining.

Generation of BT-474 and MCF7 cells in which GJB2 was knocked down using shRNA. To generate cells in which GJB2 was knocked down, the BT-474 and MCF7 cells were transfected with a shRNA construct targeting GJB2 (shGJB2) or non-targeting shRNA vectors (control shRNA; Origene, Rockville, Maryland, USA) using Lipofectamine 2000 (Invitrogen). Furthermore, the stable transfected cells were generated by selection with puromycin $(0.5 \mu \mathrm{g} / \mathrm{ml}$; Sigma-Aldrich) followed by flow cytometry-based sorting (MoFlo ${ }^{\mathrm{TM}}$ XDP; Beckman Coulter, Inc., Brea, CA, USA) for GFP expression encoded by the vector, and then were expanded and frozen for future use. Knockdown was confirmed by western blot analysis and RT-qPCR analysis.

Western blot analysis. Cell lysates were prepared using lysis buffer $50 \mathrm{mM}$ Tris- $\mathrm{HCl}$ (pH 7.4) with $1 \%$ NP40 detergent, $0.5 \%$ sodium deoxycholate, $0.1 \% \mathrm{SDS}, 1 \mathrm{mM}$ sodium fluoride, $1 \mathrm{mM}$ sodium orthovanadate, $150 \mathrm{mM}$ sodium chloride, $1 \mathrm{mM}$ EDTA (all from Sigma-Aldrich) and protease inhibitors (Calbiochem; Merck KGaA, Darmstadt, Germany). Protein estimation was performed using Bradford reagent (Sigma-Aldrich) and equal amount of protein was resolved by SDS-PAGE (12\% gel) using Bio-Rad apparatus (Bio-Rad, Hercules, CA, USA), transferred to PVDF membranes (Millipore, Billerica, MA, USA). The blot was blocked using 5\% skimmed milk (Sigma-Aldrich) as blocking reagent for $1 \mathrm{~h}$ at room temperature and probed with goat polyclonal anti-GJB2 antibody (1:1,000 dilution; ab59020; Abcam), followed by incubation with HRP-coupled rabbit anti-goat secondary antibody (1:2,000 dilution; A5420; 
Sigma-Aldrich). Immunoblots were visualized using femtoLUCENT PLUS-HRP kit (G-Biosciences, St. Louis, MO, USA). The expression of $\beta$-actin (using mouse monoclonal antibody from Sigma-Aldrich; A544; 1:2,000 dilution) was used as the loading control in all western blot analyses.

Migration assay. BT474 and MCF7 cells transfected with shGJB2 or control shRNA were seeded in 6-well Petri plates. Following $12 \mathrm{~h}$ of seeding, the cells were treated with $10 \mu \mathrm{g} / \mathrm{ml}$ of mitomycin C (Calbiochem, Merck KGaA) in serum-free medium for $2 \mathrm{~h}$ to arrest proliferation and then, two wounds were made using a P200 pipette tip. Thereafter, the cells were cultured for $48 \mathrm{~h}$ in culture medium with or without $10 \%$ FBS. Photomicrographs were captured using a microscope (Carl Zeiss, Jena, Germany) at 0 and $48 \mathrm{~h}$ after the wound generation. The distance migrated was quantified using ImageJ capture software (National Institutes of Health, Bethesda, MA, USA) and plotted as a difference of wound width between 0 and $48 \mathrm{~h}$.

Proliferation assay and cell cycle analysis. Cell proliferation was estimated by MTT assay which was performed in triplicate in 96-well plates (Nunc, Roskilde, Denmark) using BT474 and MCF7 cells stably transfected with shGJB2 or control shRNA. MTT assay was performed every $24 \mathrm{~h}$ up to 4 days. Briefly, MTT $(5 \mathrm{mg} / \mathrm{ml})$ reagent (Sigma-Aldrich) was added to each well and the plate was incubated for $4 \mathrm{~h}$ until the formazan crystals were formed. Crystals were dissolved in DMSO and the plate was read in ELISA reader at $575 \mathrm{~nm}$. Cell proliferation was expressed by plotting the absorbance of both shGJB2 transfected cells, and control shRNA transfected cells.

For cell cycle analysis, thje BT-474 and MCF7 cells transfected with control shRNA or shGJB2 $\left(5 \times 10^{5}\right)$ were seeded into 6-well plates and following $48 \mathrm{~h}$ of culturing, the cells were trypsinized and $5 \times 10^{5}$ cells were fixed by the addition of $70 \%$ ethanol overnight at $-20^{\circ} \mathrm{C}$. The cells were then washed twice with PBS, and treated with RNAse A $\left(100 \mu \mathrm{g} / \mathrm{ml}\right.$; Sigma-Aldrich) in PBS and incubated at $37^{\circ} \mathrm{C}$ for $4 \mathrm{~h}$. Furthermore, the cells were washed twice with PBS, re-suspended in $300 \mu 1$ of PBS containing propidium iodide $(20 \mu \mathrm{g} / \mathrm{ml}$; Sigma-Aldrich) and analyzed in a flow cytometer (BD FACSAria; Becton-Dickinson, Oakville, ON, Canada).

In vivo tumor formation assay. Animal experiments were performed following the approval from the Institutional Animal Ethics Committee, Indian Institute of Science (Bangalore, India). Four- to five-week-old female athymic nude mice were used for the in vivo animal experiments $(\mathrm{n}=14$; weight prior to experimentation, 15-18 g; weight upon sacrifice, 28-30 g). The animals were housed under specific pathogen-free conditions (temperature of $22 \pm 2^{\circ} \mathrm{C}$; humidity of $30-70 \%$; 12 -h light/dark cycle and fed standard sterile mouse chow and had access to sterile water ad libitum). Mice were divided into two groups ( $\mathrm{n}=7$ per group). The mice were injected with shGJB2-transfected BT474 cells or control shRNA-transfected BT474 cells $\left(10^{7}\right.$ cells) into the 5th mammary fat pad. Once the tumor attained a volume of $100 \mathrm{~mm}^{3}$, tumor size was measured regularly (each week) with digital vernier callipers. After 8 weeks, the tumors were dissected out and the animals were re-sutured and the experiment was continued for 5 more weeks to examine metastasis to other organs. The maximum tumor diameter observed in a single tumor in this study was $1.8 \mathrm{~cm}$. The dissected tumors were subjected to RNA isolation and RT-qPCR analysis for GJB2 to confirm the knockdown. Part of the tumor was processed for immunohistochemical analysis for MIB-1 (1:100 dilution; cat. no. M7240; Dako, Glostrup, Denmark) as described above to estimate the proliferation index.

Statistical analysis. Statistical analysis was performed using a paired Student's t-test for data displayed in Fig. 1A; ANOVA followed by Tukey's post hoc test for data in Fig. 1D and an unpaired Student's t-test for data analysis of all other figures. GraphPad prism version 5 software (GraphPad Software, Inc., La Jolla, CA, USA) was used for all statistical tests and plotting of the graphs. The results are presented as the means \pm SEM.

\section{Results}

GJB2 gene is upregulated in breast cancer tissues. Previous microarray data from our laboratory (30) on human breast cancer tissues and normal breast tissues (GEO Accession no. GSE40206) revealed the upregulation of the GJB2 gene in various categories of breast cancer. In this study, we validated these data using RT-qPCR analysis from the RNA isolated from breast cancer tissues and adjacent normal breast tissues. The results of RT-qPCR confirmed the upregulation of the GJB2 gene in the breast cancer tissues. GJB2 was significantly upregulated in the invasive ductal carcinoma samples $(n=50)$ when compared with the normal tissue samples $(n=25$; median $\log 2$ fold change of 4.1 ; $\mathrm{P}<0.001)$ (Fig. 1A).

GJB2 protein expression is negatively associated with the estrogen receptor status in breast cancer tissues. Since we observed an increased expression of GJB2 gene in breast cancer tissues in the above-mentioned result, we examined the expression of GJB2 protein in a total of 49 grade III invasive ductal carcinoma tissue samples (27 ER-negative and 22 ER-positive) and 21 normal breast tissue samples using immunohistochemistry. A representative staining pattern of GJB2 in normal and breast cancer tissues is depicted in Fig. 1B. The analysis revealed intense cytoplasmic and membrane staining. The staining for GJB2 was more intense in the majority of the breast cancer tissues compared with the normal breast tissue samples. Notably, we observed that the staining for GJB2 was more intense (6-9 score) in the majority of the ER-negative breast cancer tissues (19 of 27 samples, $70 \%$ ) whereas, the majority of the ER-positive breast cancer samples (17 of 22 samples, $77 \%$ ) exhibited weak to moderate staining (score $<6$ ) (Fig. 1C and D). In addition, the majority of the normal breast tissue samples exhibited weak to moderate staining. No significant association between the expression of GJB2 and HER2 or the triple-negative status was observed. The scoring of different samples used for the analysis is shown in Table I. RT-qPCR analysis of the expression of GJB2 in normal, ER-negative and ER-positive breast tumour samples revealed no association between the expression of GJB2 and ER status (data not shown). We also performed experiments in which breast cancer cells (MCF7 and BT474) were 
Table I. Immunohistochemistry of GJB2.

\begin{tabular}{|c|c|c|c|c|c|c|c|c|c|c|c|}
\hline \multicolumn{4}{|c|}{ ER-negative } & \multicolumn{4}{|c|}{ ER-positive } & \multicolumn{4}{|c|}{ Normal } \\
\hline Patient no. & Intensity & $\begin{array}{c}\text { Cells +ve } \\
(\%)\end{array}$ & Score & Patient no. & Intensity & $\begin{array}{c}\text { Cells +ve } \\
(\%)\end{array}$ & Score & Patient no. & Intensity & $\begin{array}{c}\text { Cells +ve } \\
(\%)\end{array}$ & Score \\
\hline 1 & 3 & 75 & 9 & 1 & $-\mathrm{ve}$ & & & 1 & 2 & 60 & 6 \\
\hline 2 & 3 & 60 & 9 & 2 & -ve & & & 2 & 3 & 70 & 9 \\
\hline 3 & 3 & 50 & 6 & 3 & 2 & 40 & 4 & 3 & 1 & 70 & 3 \\
\hline 4 & 2 & 20 & 4 & 4 & -ve & & & 4 & 3 & 70 & 3 \\
\hline 5 & 3 & 70 & 9 & 5 & 2 & 30 & 4 & 5 & 2 & 70 & 6 \\
\hline 6 & $-\mathrm{ve}$ & & & 6 & -ve & & & 6 & 1 & 60 & 3 \\
\hline 7 & 3 & 70 & 9 & 7 & $-\mathrm{ve}$ & & & 7 & 1 & 40 & 2 \\
\hline 8 & -ve & & & 8 & 2 & 10 & 2 & 8 & 1 & 50 & 2 \\
\hline 9 & 2 & 20 & 4 & 9 & 1 & 20 & 2 & 9 & 1 & 90 & 3 \\
\hline 10 & 3 & 70 & 9 & 10 & 2 & 75 & 6 & 10 & 1 & 90 & 3 \\
\hline 11 & 2 & 35 & 4 & 11 & 2 & 80 & 6 & 11 & 2 & 60 & 6 \\
\hline 12 & 2 & 30 & 4 & 12 & 2 & 80 & 6 & 12 & 2 & 65 & 6 \\
\hline 13 & 2 & 30 & 4 & 13 & 2 & 80 & 6 & 13 & 1 & 10 & 1 \\
\hline 14 & 3 & 50 & 6 & 14 & 3 & 80 & 9 & 14 & 1 & 10 & 1 \\
\hline 15 & 3 & 80 & 9 & 15 & 1 & 60 & 3 & 15 & 1 & 50 & 3 \\
\hline 16 & 3 & 60 & 9 & 16 & 1 & 60 & 3 & 16 & 1 & 30 & 2 \\
\hline 17 & 3 & 65 & 9 & 17 & 2 & 40 & 4 & 17 & 1 & 20 & 2 \\
\hline 18 & 3 & 90 & 9 & 18 & -ve & & & 18 & 3 & 80 & 9 \\
\hline 19 & 3 & 60 & 9 & 19 & 1 & 45 & 2 & 19 & 1 & 50 & 2 \\
\hline 20 & 3 & 75 & 9 & 20 & 2 & 45 & 4 & 20 & 1 & 10 & 2 \\
\hline 21 & 3 & 60 & 9 & 21 & 2 & 20 & 2 & 21 & 1 & 80 & 3 \\
\hline 22 & 3 & 75 & 9 & 22 & -ve & & & & & & \\
\hline 23 & -ve & & & & & & & & & & \\
\hline 24 & 3 & 70 & 9 & & & & & & & & \\
\hline 25 & 3 & 40 & 6 & & & & & & & & \\
\hline 26 & 3 & 75 & 9 & & & & & & & & \\
\hline 27 & 3 & 80 & 9 & & & & & & & & \\
\hline
\end{tabular}

Scoring and quantification was performed in normal tissues, ER-positive (+ve) and ER-negative (-ve) breast tumor tissues as described in the Materials and methods.; ER, estrogen receptor; PR, progesterone receptor.

treated with estradiol and assessed the expression of GJB2. However, no regulation of GJB2 by estradiol was observed (data not shown).

Knockdown of GJB2 promotes the migration and inhibits the proliferation of breast cancer cells. Since the above-mentioned results revealed high levels of GJB2 in breast cancer tissues, we further investigated the role of GJB2 in breast cancer cell migration and proliferation. We generated BT474 and MCF7 breast cancer cells in which GJB2 was knocked down using shRNA in (Fig. 2). We then performed a scratch assay with the BT474 and MCF7 cells stably transfected with shGJB2. The shGJB2-transfected BT474 and MCF7 cells demonstrated a significant increase in migration (80\% in MCF7 cells; Fig. 3A and C; and 38\% in BT474 cells; Fig. 3B and D), when compared to the control shRNA-transfected cells $(30 \%$ in MCF7 cells and 28\% in BT474 cells) after $48 \mathrm{~h}$.
To further investigate the functional link between the proliferation of cancer cells and GJB2, we performed a proliferation assay and analyzed the doubling time for the control shRNA-transfected cells the cells in which GJB2 was knocked down. We observed that the knockdown of GJB2 significantly decreased the proliferation of the cells at all time-points $(\mathrm{P}<0.001$; Fig. $4 \mathrm{~A})$. We also observed that the doubling time was increased (data not shown) in the shGJB2-transfected cells $(40.02 \pm 2.85 \mathrm{~h}$ in BT474 cells and $54.65 \pm 1.98 \mathrm{~h}$ in MCF7 cells) compared to the control shRNA-transfected cells $(29.79 \pm 1.9 \mathrm{~h}$ in BT474 cells and $35.43 \pm 2.6 \mathrm{~h}$ in MCF7 cells). Furthermore, we also performed a cell cycle analysis to determine the effects of the knockdown of GJB2 on the cell cycle profile. Cell cycle analysis did not reveal any significant difference in any of the cell cycle phases (G1, S and G2/M) between the control shRNA-transfected and the cells in which GJB2 was knocked down (Fig. 4B). 


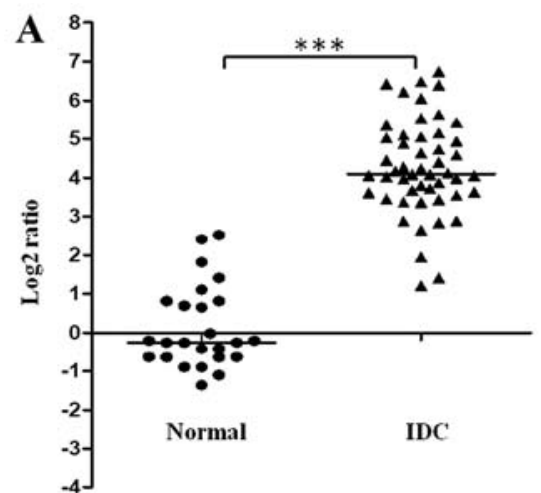

B

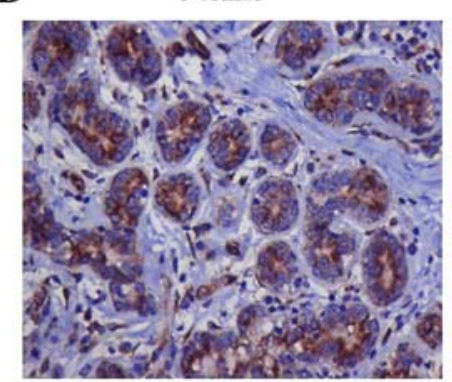

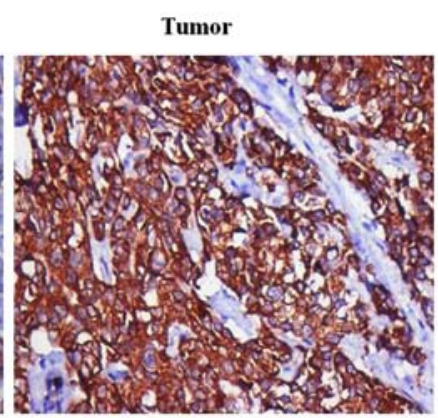

C

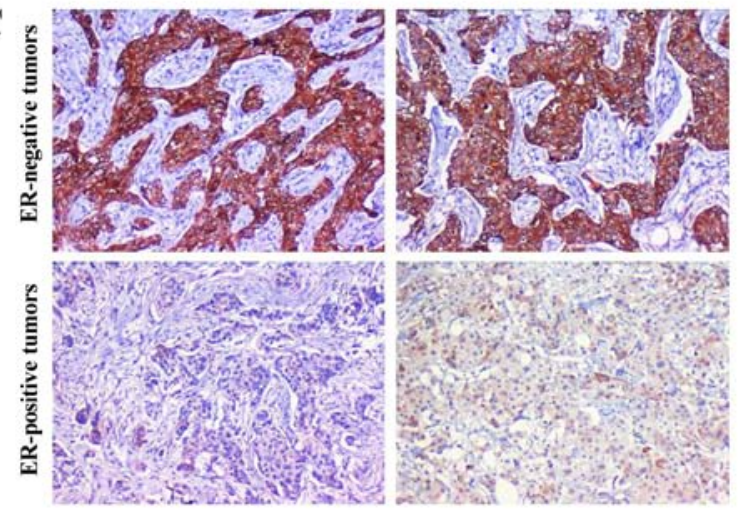

D

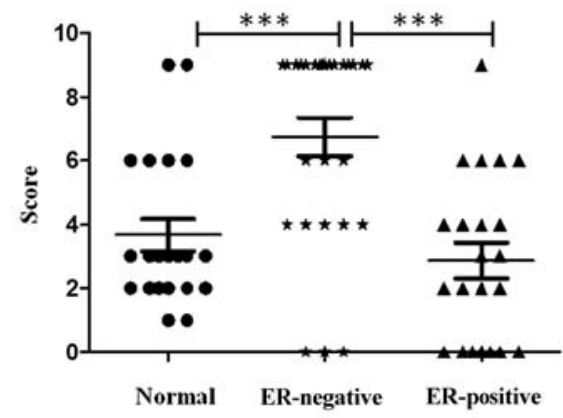

Figure 1.Expression of GJB2 in breast cancer tissue samples.(A) Relative mRNA expression of GJB2 in normal and breast cancer tissue samples.(B) Representative images of immunohistochemical expression of GJB2 protein in normal and breast cancer tissue samples (magnification, x20). (C) Representative images of GJB2 protein expression in ER-negative and ER-positive breast cancer tissue samples (magnification, x10) and (D) relevant quantification. Each experiment was performed in triplicate and error bar represents the standard error of the mean $(\mathrm{SEM}) .{ }^{* * * *} \mathrm{P}<0.001$.

A

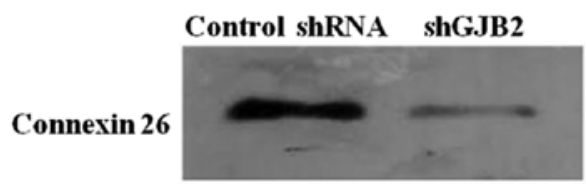

p-actin

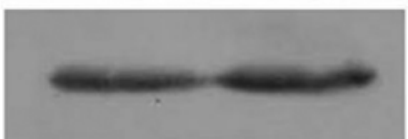

B

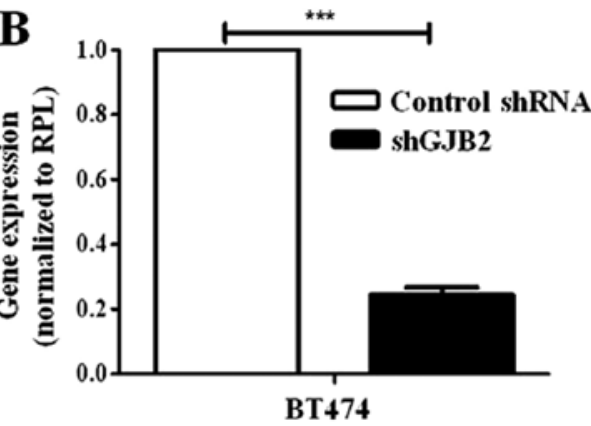

MCF7
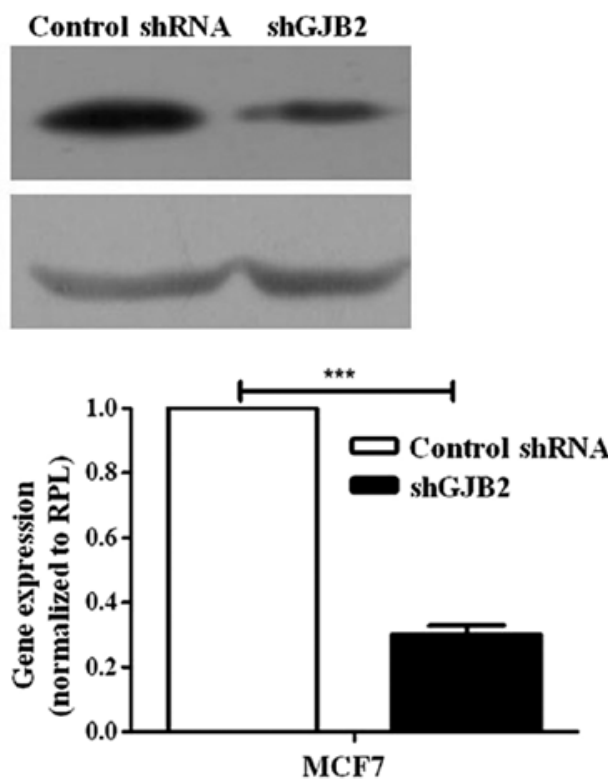

Figure 2. Knockdown of GJB2 in BT474 breast cancer cell lines. (A) Protein lysate from cells (BT474 and MCF7) transfected with control shRNA and shGJB2 were subjected to western blot analysis to confirm the knockdown of GJB2 protein. $\beta$-actin was used as a normalizing control. (B) Real-time PCR analysis for the expression of GJB2 to confirm the knockdown in BT474 and MCF7 cells. Each experiment was performed in triplicate (n=3) and error bars represent the means \pm standard error of the mean $(\mathrm{SEM}) .{ }^{* * *} \mathrm{P}<0.001$.

Knockdown of GJB2 suppresses tumor formation and the proliferation of breast tumor cells in vivo. To further confirm the above-mentioned results in vivo, we performed an orthotopic xenograft tumor formation assay using immunocompromised mice. Consistent with the above-mentioned in vitro data, we observed a significant reduction in the growth 

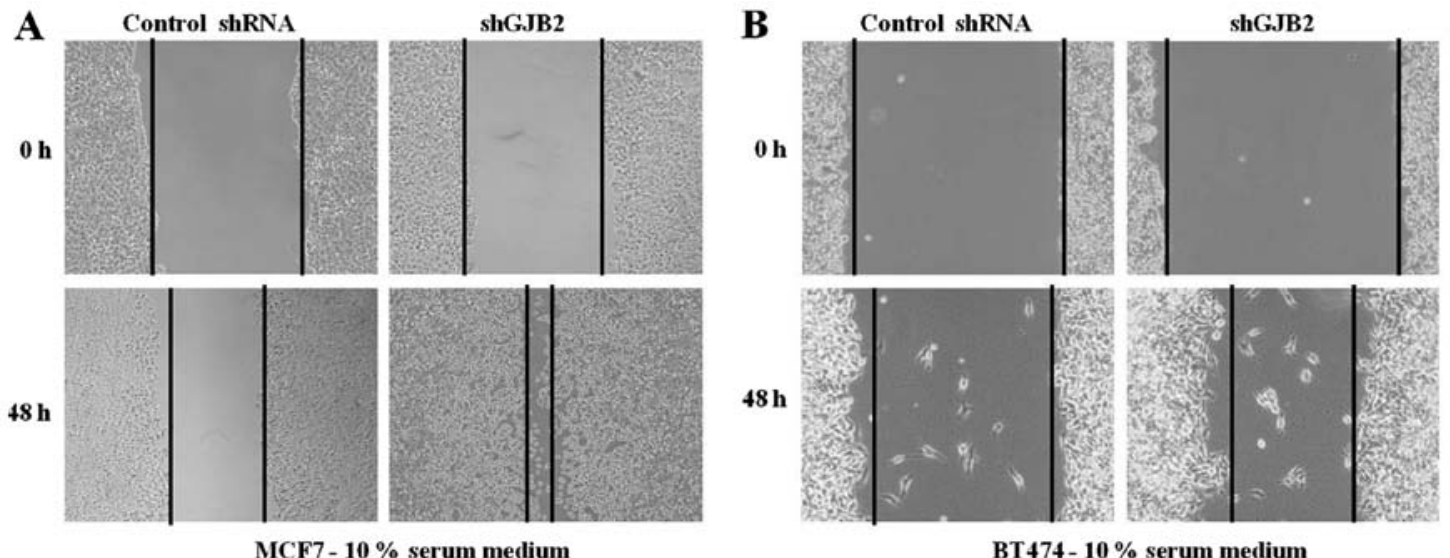

BT474- $10 \%$ serum medium
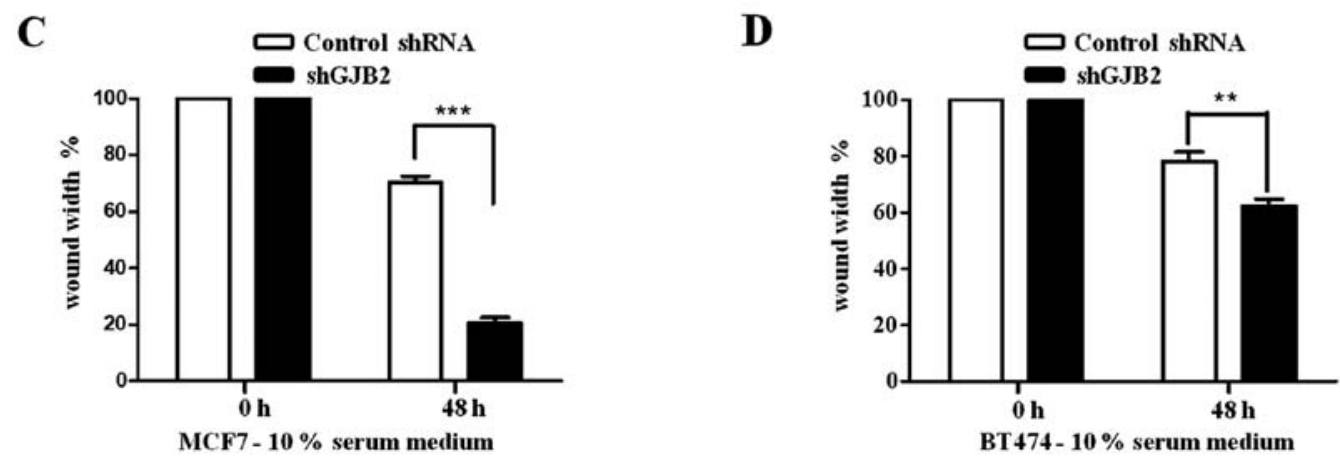

Figure 3. Effect of GJB2 on the migration of BT474 and MCF7 breast cancer cell lines. (A and B) Representative images of control shRNA- and shGJB2transfected MCF7 and BT474 cells after 0 and $48 \mathrm{~h}$ of the scratch in medium containing $10 \%$ serum. (C and D) Quantification of the migration assay by ImageJ software. The assay was performed three times $(\mathrm{n}=3)$ and error bars represent the means \pm standard error of the mean $(\mathrm{SEM}){ }^{* * *} \mathrm{P}<0.01$ and ${ }^{* * * *} \mathrm{P}<0.001$.
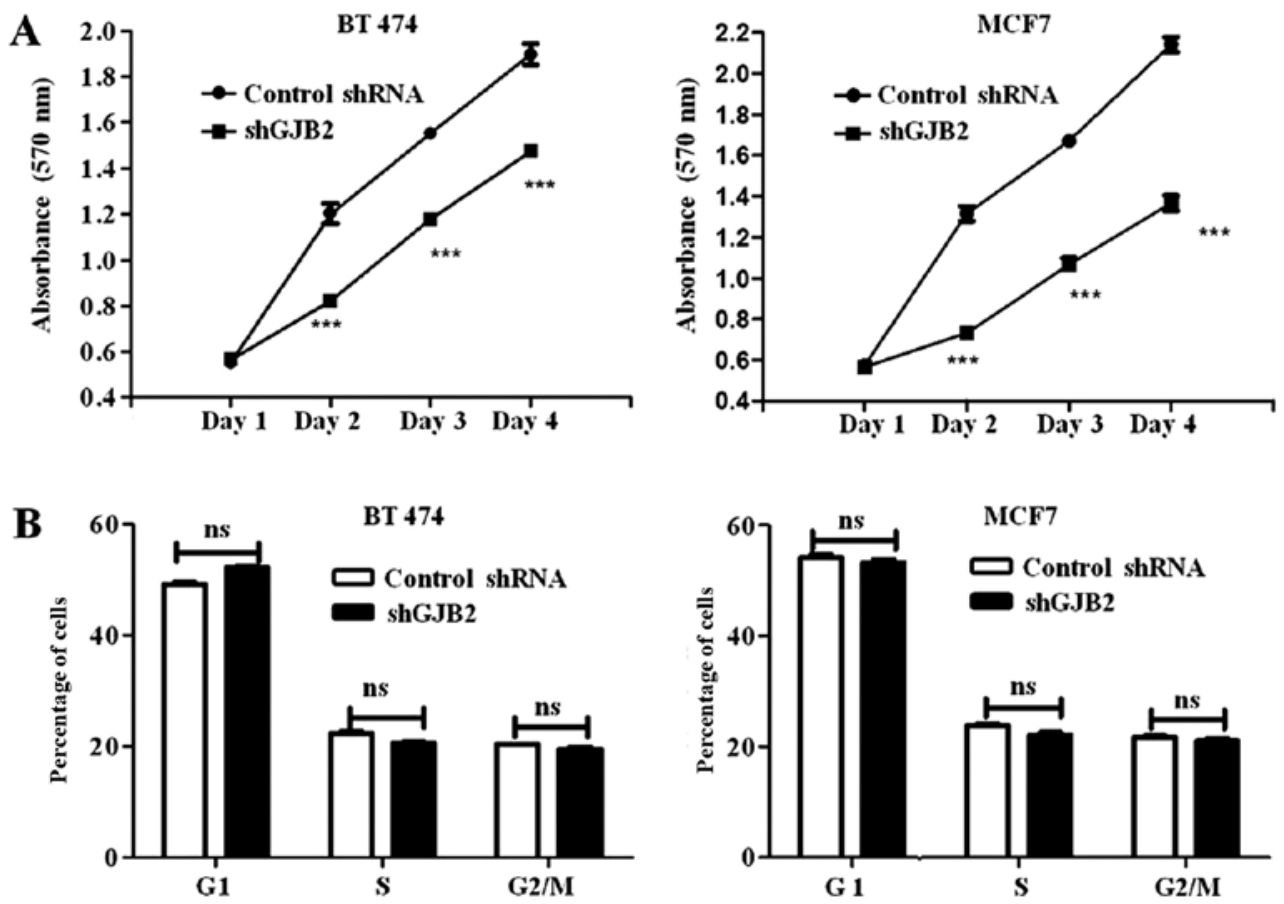

Figure 4. Effect of GJB2 knockdown on the proliferation of BT474 and MCF7 cells. (A) Control shRNA- and shGJB2-transfected BT474 and MCF7 cells were cultured in 96-well plates and MTT assay was performed each day up to four days; absorbance was recorded at $570 \mathrm{~nm}$. (B) Flow cytometric cell cycle analysis of control shRNA- and shGJB2-transfected BT474 and MCF7 cells. The percentage of cells in each phase of the cell cycle (G1, S and G2/M) is indicated in the $y$ axis of the graph. Both assays were performed in triplicate and three times $(n=3)$. Error bar represents the means \pm standard error of the mean $(\mathrm{SEM})$. ${ }^{* * * *} \mathrm{P}<0.001$ vs. control shRNA-transfected cells; ns, not significant.

of tumors derived from cells in which GJB2 was knocked down (49.5\%; $\mathrm{P}<0.001)$ compared to the tumors derived from control
shRNA-transfected cells (Fig. 5A). We also confirmed the knockdown of GJB2 in tumors by subjecting the RNA isolated 
A

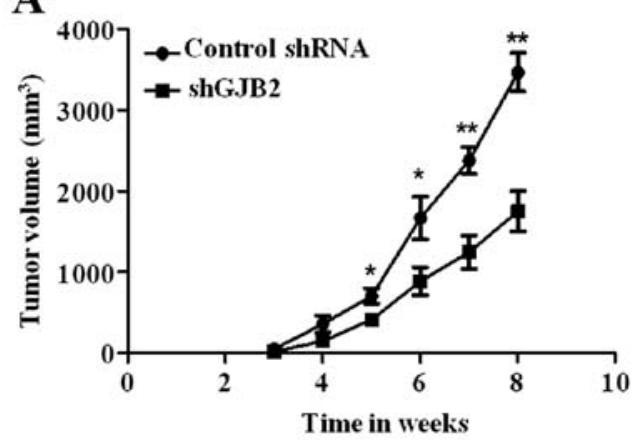

C
B

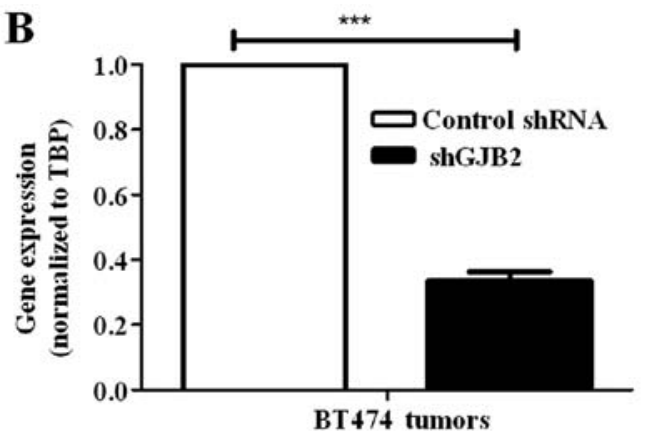

Figure 5. GJB2 knockdown reduces tumor growth and proliferation in vivo. (A) The line graph shows the tumor volume of the xenograft tumors in nude mice $(n=7)$ derived from control shRNA- and shGJB2-transfected BT474 cells. (B) RNA isolated from tumors derived from control shRNA- and shGJB2-transfected BT474 cells was subjected to RT-qPCR analysis to confirm the knockdown of GJB2. (C) Representative images of immunohistochemical staining for MIB-1 to examine the proliferation in the tumor sections derived from control shRNA- and shGJB2-transfected BT474 cells ( $\mathrm{n}=7$, magnification, $\mathrm{x} 20$ ). Error bar represents the means \pm standard error of the mean $(\mathrm{SEM}) .{ }^{*} \mathrm{P}<0.05,{ }^{* *} \mathrm{P}<0.01$ and ${ }^{* * *} \mathrm{P}<0.001$ vs. control.

from tumors to RT-qPCR analysis (Fig. 5B). Furthermore, we also performed immunohistochemistry for MIB-1 to examine the proliferation rate in these tumors. There was a significant decrease in the proliferation rate in the tumors derived from cells in which GJB2 was knocked down (almost 50\%) compared to the tumors derived from control shRNA-transfected cells (Fig. 5C). There was no observable metastasis of both the tumors derived from shGJB2-transfected cells and those derived from control shRNA-transfected cells to any other organs (data not shown).

\section{Discussion}

Previous studies have revealed that in some types of cancer, some of the connexins and other gap junction proteins are downregulated (31-33). However, GJB2 (connexin 26) overexpression has been reported to be expressed in various carcinomas, including breast cancer $(17,18,21,24-26)$. Our data derived from both RT-qPCR and immunohistochemical analysis of human breast cancer tissue samples confirmed these findings. In addition, our analysis revealed a negative association of GJB2 protein expression with the ER status of breast cancer tissue samples. Little is known about the regulation of connexins by estrogen. However, Saito et al (34) demonstrated that the activation of ER- $\alpha$ by estrogen resulted in the downregulation of connexins, leading to the suppression of gap junctional intercellular communication and the promotion of tumor progression in endometrial carcinoma. In addition, a recent study indicated that other forms of connexions, such as connexin 43 are regulated by estradiol in glioma cell lines (35). Hence, we hypothesized that GJB2 protein may also be regulated by estrogen receptor in breast cancers.
Notably, we did not observe the regulation of GJB2 RNA by the estrogen treatment of breast cancer cells (data not shown). In addition, the association between GJB2 and the ER status was observed only in tumor tissues by immunohistochemistry. However, no differences in the RNA expression of GJB2 in ER-positive and -nevative breast cancer tissues were observed (data not shown). This could be attributed to the post-transcriptional regulation of GJB2, which may be indirectly associated with the ER status of the tumors. Furthermore, breast cancer cells treated with estradiol did not exhibit evidence of GJB2 regulation (data not shown). This indicated that other variables, including the tumor microenvironment may be involved in the regulation of GJB2, which requires further investigations. However, the negative association of GJB2 protein with the ER status in our study indicated an alternate mechanism of GJB2 regulation by ER, which is not yet understood. Further studies are warranted in order to elucidate the mechanisms of GJB2 regulation in ER-negative breast tumors.

It is well known that gap junctional intercellular communication (GJIC), mediated by connexins play an important role in normal mammary gland development and homeostasis, and also in the progression of breast cancer (15). In line with this, our study revealed that the knockdown of GJB2 promoted the migration and inhibited the proliferation of both breast cancer cell lines, which are ER-positive. This result suggested a role for GJB2 in the inhibition of the migration of ER-positive cells. This is of importance, since ER-positive tumors exhibit the marginal expression of GJB2, almost similar to normal tissues. Hence, it is likely that ER-positive tumors have a greater migratory capacity than ER-negative tumors. Further studies on a larger cohort of patients are required in 
order to establish an association between GJB2 and tumor cell migration. In addition, tumors in immunocompromised mice formed from cells in which GJB2 was knocked down were significantly smaller compared to those derived from GJB2-expressing cells, suggesting a growth advantage when GJB2 is overexpressed. The lack of GJIC due to the loss of connexins during cancer progression may allow tumor cells to physically detach from the microenvironment and migrate. Our data revealed the increased expression of GJB2 in the majority of the IDC (grade 3) breast tissues, which are highly proliferative. In agreement with this, our data on the knockdown of GJB2 in breast cancer cells revealed significant decrease in the proliferation of cells, as well as tumour growth in immunocompromised mice. Hence, it is clear from the available studies, as well as from our results that depending on the stages of breast cancer, GJB2 can act as tumor suppressor or promote tumor growth.

In conclusion, in the present study, we found that GJB2 was overexpressed in invasive breast cancer tissue samples compared to normal breast tissues and the expression of GJB2 was associated with the ER status. In addition, the knockdown of GJB2 in breast cancer cells affected the migration properties of the breast cancer cells. Furthermore, GJB2 knockdown reduced the proliferation of the breast cancer cells in vitro and in vivo. Hence, GJB2 appears to be an important regulator of breast tumorigenesis. Further studies are warranted in order to elucidate the exact role of GJB2 in breast tumorigenesis.

\section{Acknowledgements}

The authors are grateful to Dr Vani Santosh for assisting with the immunohistochemistry of mouse tumors. In addition, the authors are grateful for the infrastructure support to the Department of Molecular Reproduction, Development and Genetics (MRDG) and the Indian Institute of Science (IISc) by the DST-FIST, the UGC and the DBT-IISc partnership. The authors also acknowledge the flow cytometric and animal facility at the Indian Institute of Science (IISc) for their support with the experiments.

\section{Funding}

The present study received funding by the Department of Biotechnology (DBT) in a program support and fellowship to SD. AS received funding from UGC, Government of India for D.S Kothari postdoctoral fellowship.

\section{Availability of data and materials}

All data generated or analyzed during this study are included in this published article.

\section{Authors' contributions}

AS, design and execution of experiments, analysis of data and manuscript writing; SD, tumor tissue data acquisition and realtime PCR analysis; GM, immunohistochemistry and pathological evaluation; PK, conceived, guidance, data analysis and manuscript writing. All authors read and approved the manuscript, and agree to be accountable for all aspects of the research in ensuring that the accuracy or integrity of any part of the work are appropriately investigated and resolved.

\section{Ethics approval and consent to participate}

For the use of patient samples, all patients provided informed consent following the approval of the KMIO Medical Ethics Committee. Animal experiments were performed with the approval from the Institutional Animal Ethics Committee, Indian Institute of Science (Bangalore, India).

\section{Patient consent for publication}

Not applicable.

\section{Competing interests}

The authors declare that they have no competing interests.

\section{References}

1. Charles AC, Naus CC, Zhu D, Kidder GM, Dirksen ER and Sanderson MJ: Intercellular calcium signaling via gap junctions in glioma cells. J Cell Biol 118: 195-201, 1992.

2. Monaghan $P$ and Moss D: Connexin expression and gap junctions in the mammary gland. Cell Biol 20: 121-125, 1996.

3. Goodenough DA, Goliger JA and Paul DL: Connexins, connexons, and intercellular communication. Annu Rev Biochem 65: 475-502, 1996.

4. Zhu D, Caveney S, Kidder GM and Naus CC: Transfection of C6 glioma cells with connexin $43 \mathrm{cDNA}$ : Analysis of expression, intercellular coupling, and cell proliferation. Proc Natl Acad Sci USA 88: 1883-1887, 1991.

5. Naus CC, Elisevich K, Zhu D, Belliveau DJ and Del Maestro RF: In vivo growth of C6 glioma cells transfected with connexin43 cDNA. Cancer Res 52: 4208-4213, 1992.

6. Eghbali B, Kessler JA, Reid LM, Roy C and Spray DC: Involvement of gap junctions in tumorigenesis: Transfection of tumor cells with connexin $32 \mathrm{cDNA}$ retards growth in vivo. Proc Natl Acad Sci USA 88: 10701-10705, 1991.

7. Aasen T: Connexins: Junctional and non-junctional modulators of proliferation. Cell Tissue Res 360: 685-699, 2015.

8. Carette D, Gilleron J, Chevallier D, Segretain D and Pointis G: Connexin a check-point component of cell apoptosis in normal and physiopathological conditions. Biochimie 101: 1-9, 2014.

9. King TJ and Bertram JS: Connexins as targets for cancer chemoprevention and chemotherapy. Biochim Biophys Acta 1719: 146-160, 2005.

10. Kotini $M$ and Mayor R: Connexins in migration during development and cancer. Dev Biol 401: 143-151, 2015.

11. Defamie N, Chepied A and Mesnil M: Connexins, gap junctions and tissue invasion. FEBS Lett 588: 1331-1338, 2014.

12. McLachlan E, Shao Q, Wang HL, Langlois S and Laird DW: Connexins act as tumor suppressors in three-dimensional mammary cell organoids by regulating differentiation and angiogenesis. Cancer Res 66: 9886-9894, 2006.

13. Wang WK Chen MC, Leong HF, Kuo YL, Kuo CY and Lee CH: Connexin 43 suppresses tumor angiogenesis by down-regulation of vascular endothelial growth factor via hypoxic-induced factor-1 $\alpha$. Int J Mol Sci 16: 439-451, 2015.

14. Aasen T, Mesnil M, Naus CC, Lampe PD and Laird DW: Gap junctions and cancer: Communicating for 50 years. Nat Rev Cancer 16: 775-788, 2016.

15. Banerjee D: Connexin's connection in breast cancer growth and progression. Int J Cell Biol 2016: 9025905, 2016.

16. Kelsell DP, Dunlop J, Stevens HP, Lench NJ, Liang JN, Parry G, Mueller RF and Leigh IM: Connexin 26 mutations in hereditary non-syndromic sensorineural deafness. Nature 387: 80-83, 1997.

17. Pfeffer F, Koczan D, Adam U, Benz S, von Dobschuetz E, Prall F, Nizze H, Thiesen HJ, Hopt UT and Löbler M: Expression of connexin 26 in islets of Langerhans is associated with impaired glucose tolerance in patients with pancreatic adenocarcinoma. Pancreas 29: 284-290, 2004. 
18. Villaret DB, Wang T, Dillon D, Xu J, Sivam D, Cheever MA and Reed SG: Identification of genes overexpressed in head and neck squamous cell carcinoma using a combination of complementary DNA subtraction and microarray analysis. Laryngoscope 110 : 374-81, 2000.

19. Kanczuga-Koda L, Sulkowski S, Koda M and Sulkowska M: Alterations in connexin26 expression during colorectal carcinogenesis. Oncology 68: 217-222, 2005.

20. Tate AW, Lung T, Radhakrishnan A, Lim SD, Lin X and Edlund M: Changes in gap junctional connexin isoforms during prostate cancer progression. Prostate 66: 19-31, 2006.

21. Haass NK, Wladykowski E, Kief S, Moll I and Brandner JM: Differential induction of connexins 26 and 30 in skin tumors and their adjacent epidermis. J Histochem Cytochem 54: 171-182, 2006.

22. Tan LW, Bianco T and Dobrovic A: Variable promoter region $\mathrm{CpG}$ island methylation of the putative tumor suppressor gene connexin 26 in breast cancer. Carcinogenesis 23: 231-236, 2002.

23. Jamieson S, Going JJ, D'Arcy R and George WD: Expression of gap junction proteins connexin 26 and connexin 43 in normal human breast and in breast tumours. J Pathol 184: 37-43, 1998.

24. Naoi Y, Miyoshi Y, Taguchi T, Kim SJ, Arai T, Tamaki Y and Noguchi S: Connexin26 expression is associated with lymphatic vessel invasion and poor prognosis in human breast cancer. Breast Cancer Res Treat 106: 11-17, 2007.

25. Ito A, Koma Y, Uchino K, Okada T, Ohbayashi C, Tsubota N and Okada M: Increased expression of connexin 26 in the invasive component of lung squamous cell carcinoma: Significant correlation with poor prognosis. Cancer Lett 234: 239-248, 2006.

26. Inose T, Kato H, Kimura H, Faried A, Tanaka N, Sakai M, Sano A, Sohda M, Nakajima M, Fukai, et al: Correlation between connexin 26 expression and poor prognosis of esophageal squamous cell carcinoma. Ann Surg Oncol 16: 1704-1710, 2009.
27. Teleki I, Krenacs T, Szasz MA, Kulka J, Wichmann B, Leo C, Papassotiropoulos B, Riemenschnitter C, Moch H and Varga Z: The potential prognostic value of connexin 26 and 46 expression in neoadjuvant treated breast cancer. BMC Cancer 13: 50, 2013.

28. Livak KJ and Schmittgen TD: Analysis of relative gene expression data using real time quantitative PCR and the $2^{-\Delta \Delta C_{\mathrm{T}}}$ method. Methods 25: 402-408, 2001.

29. Sehgal P, Kumar N, Praveen Kumar VR, Patil S, Bhattacharya A, Vijaya Kumar M, Mukherjee G and Kondaiah P: Regulation of protumorigenic pathways by insulin like growth factor binding protein 2 and its association along with $\beta$-catenin in breast cancer lymph node metastasis. Mol Cancer 12: 63, 2013.

30. Bashir M, Damineni S, Mukhrejee G and Kondaiah P: Actavin-A signalling promotes epithelial-mesenchymal transition, invasion, and metastatic growth of breast cancer. NPJ Breast Cancer 1: 15007, 2015.

31. Lee SW, Tomasetto C, Paul D, Keyomarsi K and Sager R: Transcriptional downregulation of gap-junction proteins blocks junctional communication in human mammary tumor cell lines. J Cell Biol 118: 1213-1221, 1992.

32. Janssen-Timmen U, Traub O, Dermietzel R, Rabes HM and Willecke K: Reduced number of gap junctions in rat hepatocarcinomas detected by monoclonal antibody. Carcinogenesis 7: 1475-1482, 1986

33. Mesnil M: Connexins and cancer. Biol Cell 94: 493-500, 2002.

34. Saito T, Tanaka R, Wataba K, Kudo R and Yamasaki H: Overexpression of estrogen receptor-alpha gene suppresses gap junctional intercellular communication in endometrial carcinoma cells. Oncogene 23: 1109-1116, 2004.

35. Moinfar Z, Dambach H, Schoenebeck B, Forster E, Prochnow N and Faustmann PM: Estradiol receptors regulate differential connexin 43 expression in F98 and C6 glioma cell lines. PLoS One 11: e0150007, 2016. 